DOI: $10.23857 /$ dc.v5i1.848

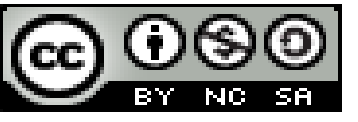

Ciencias de la salud

Artículo Original

\title{
Prevención de paciente con problemas de sedentarismo cardiovascular
}

\section{Prevention of patient with problems of cardiovascular sedentarism}

\section{Prevenção de paciente com problemas de sedentarismo cardiovascular}

\author{
Saskia J. Mendoza-García I \\ saskia.mendoza123@hotmail.com \\ Jean Carlos-Delgado II \\ carlosdelgado@gmail.com \\ Mónica C. Calderón-Moreira ${ }^{\mathrm{III}}$ \\ calderón-moni69@yahoo.es \\ Ana B. Castro-Soriano IV \\ anita789@hotmail.com \\ Felipe A. Bajaña-Andrade V \\ felipecastro@gmail.com \\ Marjorie L. Erazo-Villacreses VI \\ majo.erazo@hotmail.com
}

Recibido: 27 de octubre de 2018 * Corregido: 28 de noviembre de 2018 * Aceptado: 16 de diciembre de 2018

\footnotetext{
Medica Cirujana en el Hospital de Especialidades, Portoviejo, Ecuador.

Médico Residente Hospital Gustavo Dominguez, Santo Domingo, Ecuador.

Médico Residente Hospital Gustavo Dominguez, Santo Domingo, Ecuador.

Médico Residente Hospital Gustavo Dominguez, Santo Domingo, Ecuador.

v. Médico Residente Hospital Gustavo Dominguez, Santo Domingo, Ecuador.

VI. Médico Residente del Hospital Básico de Sangolquí, Sangolqui, Ecuador.
} 


\section{Resumen}

El sedentarismo es en la actualidad una de las principales fuentes de amenaza para la salud de las personas como efecto de la inactividad física provocando los problemas cardiovasculares, la prevención, tratamiento, observación y recuperación a causa de las enfermedades crónicas no transmisibles; es así que los especialistas en ciencias médicas y del deporte y consecuentemente las autoridades sanitarias recomiendan que toda persona con o sin problemas cardiovasculares incluyan en el desempeño de su vida cotidiana, tanto en el trabajo como en el hogar desarrollen actividad física de forma regular, ya sea para recuperarse o mantener su salud. Por lo tanto, es de considerar los beneficios de la actividad física como herramienta clave para solucionar los problemas propios del sedentarismo causando los problemas cardiovasculares, las nuevas recomendaciones de actividad física para la salud establecen con la aprobación de la OMS, destacan que al menos 30 minutos diarios, por lo menos cinco días de la semana, de forma continua o en sesiones acumulativas de 10 o 15 minutos de una actividad de intensidad moderada.

Palabras claves: Sedentarismo; Actividad Física; Prevención; Problemas Cardiovasculares; Beneficios. 


\begin{abstract}
The current sedentarism is one of the main sources of threat to the health of people as a result of physical inactivity causing cardiovascular problems, prevention, treatment, observation and recovery due to chronic noncommunicable diseases; Thus, specialists in medical sciences and sports and consequently health authorities recommend that everyone with or without cardiovascular problems include in the performance of their daily lives, both at work and at home develop physical activity on a regular basis, and either to recover or maintain your health. Therefore, it is to consider the benefits of physical activity as a key tool to solve the problems of sedentary lifestyle causing cardiovascular problems, the new recommendations of physical activity for health established with the approval of the WHO, highlight that at least 30 minutes daily, at least five days a week, continuously or in cumulative 10 or 15 minute sessions of a moderate intensity activity.
\end{abstract}

Key words: Sedentary lifestyle, physical activity, prevention, cardiovascular problems, benefits. 


\section{Introducción.}

Los beneficios preventivos del ejercicio regular dan respuestas metabólicas y hemodinámicas características de la actividad física regular que permiten prevenir el desarrollo de la enfermedad cardiovascular. La demostración epidémica y efectiva de las presentes décadas presenta con transparencia que a pesar de este conocimiento, la prevalencia del sedentarismo en el mundo actual es alarmante.

Esta situación ha convertido la vida sedentaria en un factor de riesgo con grandes implicaciones para las políticas de salud pública, y por supuesto, la prescripción y realización de actividad física, una intervención fundamental y factor protector para disminuir tanto el riesgo cardiovascular como el riesgo para otras enfermedades crónicas. Aunque no ha sido fácil obtener conclusiones contundentes acerca de la relación entre actividad física y enfermedad cardiovascular, podemos afirmar hoy en día que el ejercicio regular hace parte indiscutible de la prevención cardiovascular, por lo tanto el conocimiento sobre los principales aspectos del mismo resulta fundamental para prescribirlo e implementarlo en todos los momentos del ciclo vital y de acuerdo a situación clínica (prevención, primordial, primaria, secundaria, terciaria), Duperly y Anchique, (2014).

El análisis de los beneficios de la práctica regular de alguna actividad física y de los riesgos derivados de un estilo de vida sedentario han sido objeto de investigación en numerosos estudios epidemiológicos observacionales dada su importante contribución a la morbimortalidad, principalmente a través del incremento del riesgo cardiovascular y su relación con el síndrome metabólico. Los costes derivados de estas patologías hacen que el sedentarismo y el sobrepeso al 
que conduce puedan representar una carga económica para el sistema sanitario comparable a la derivada del hábito tabáquico o a la atribuible a los bebedores abusivos, Varo y Martínez, (2007).

Numerosos estudios epidemiológicos han constatado que la falta de actividad física y el sedentarismo afecta negativamente a la salud de la sociedad en general y de las personas mayores en particular, Varo et al., (2003).

Se habla de ejercicio físico cuando la actividad física es planificada, estructurada y repetitiva, y tiene por objeto la mejora o el mantenimiento de uno o más componentes de la forma física. El deporte es una actividad física reglamentada y competitiva. Virtualmente, todas las actividades de condicionamiento físico y la mayoría de los deportes se consideran ejercicio físico. La forma física es un conjunto de características que poseen o alcanzan los individuos en relación a la capacidad de realizar actividad física, Serra et al, (1994).

La actividad física se define como cualquier movimiento corporal producido por los músculos esqueléticos y que tiene como resultado un gasto energético que se añade al gasto del metabolismo basal. La actividad física se mide en kcal o kilojulios o consumo de oxígeno o METS (múltiples de metabolismo basal). Otra forma más burda de medirla, pero de gran utilidad en la práctica de ejercicio no competitivo, puede ser la medida de la frecuencia cardíaca, Serra et al, (1994).

La inactividad física es un importante factor de riesgo para muchas condiciones y problemas crónicos de salud, tales como las enfermedades cardiovasculares, la hipertensión, la obesidad, la osteoporosis, la diabetes mellitus y de salud mental, Christmas y Andersen, (2000). 
La enfermedad cardiovascular (ECV) es la primera causa de muerte en el mundo. En las últimas décadas, si bien el tratamiento y control de algunos factores de riesgo (FR), como la hipertensión y la dislipidemia, han mejorado, el manejo de otros FR, como el sobrepeso, obesidad y sedentarismo, ha sido menos exitoso. Acevedo et al. (2013).

Importantes avances en el tratamiento de las enfermedades cardiovasculares han sido facilitados por la identificación de los factores de riesgo tradicionales, pero a pesar de la evidencia clínica acumulada, la implementación de estrategias para prevenir las enfermedades cardiovasculares aún permanecen lejos de ser óptimas, por lo que el Médico de Familia juega un importante rol en la prevención primaria individual durante la consulta con sus pacientes, en la que se requiere de un enfoque de tratamiento individualizado, y el médico no debe centrar su intervención en un factor de riesgo específico cuando múltiples factores de riesgo cardiovascular estén presentes. Sus recomendaciones en los cambios en el estilo de vida o el tratamiento medicamentoso deberían estar basadas en la estimación del riesgo cardiovascular, Erhardt, L., (2007).

El sedentarismo ha sido identificado como factor de riesgo para enfermedades cardiovasculares. Estudios anteriores constataron que $76,9 \%$ de las personas que sufrieron infarto y $81,2 \%$ de los portadores de hipertensión arterial no realizaban ningún tipo de actividad, Simonetti et al, (2002).

La Organización Mundial de la Salud, recoge en su «Informe sobre la Salud en el mundo 2002», que los estilos de vida sedentarios son una de las diez causas fundamentales de muerte y discapacidad en el mundo, World Health Organization, (WHO, 2002). 
La Organización Mundial de la Salud (OMS, 2002), definió el sedentarismo como la ausencia de la actividad física necesaria para que el organismo humano se mantenga en un estado, saludable y lo ubica como un factor de riesgo y causa del incremento de la mortalidad, morbilidad y discapacidad en el mundo actual.

En el estudio «Global Burden of Disease Study» (estudio sobre la Carga Global de Enfermedad), se calcula que la inactividad física Número 17, 2010 (1 ${ }^{\circ}$ semestre) RETOS. Nuevas tendencias en Educación Física, Deporte y Recreación - 127 - constituye la octava causa de mortalidad en el mundo y representa un 1\% de la carga total de enfermedad Murray y López, (2002).

A pesar del preocupante aumento de la prevalencia del sedentarismo, parece que no se termina de ser consciente de la urgente necesidad de desarrollar políticas poblacionales y estrategias efectivas encaminadas a la promoción de la actividad física y a la prevención de los estilos de vida sedentarios, Varo y Martínez, (2007).

\section{Metodología.}

Para la realización del presente artículo científico se efectúo mediante un análisis de la bibliografía de la literatura más próxima sobre la prevención de paciente con problemas de sedentarismo cardiovascular. En esta investigación, se consideró, información confiable respecto a trabajos plasmados, tratados referente a este tema, se utilizo información bibliográfica, consultas electrónicas, misma que se la obtuvo de libros electrónicos, artículos científicos y revistas. 


\section{Desarrollo.}

\section{Los beneficios del ejercicio físico como medida de prevención}

Los beneficios evidentes del ejercicio físico empleados como medida de prevención primaria y secundaria en la cardiopatía isquémica se han señalado en diversas publicaciones. También se han publicado efectos favorables del entrenamiento físico empleado como parte de un programa de rehabilitación cardiaca en lo referente a calidad de vida, morbilidad y mortalidad en pacientes con enfermedad de las arterias coronarias y otras enfermedades cardiovasculares, Rivas, (2011).

Para lograr tales efectos beneficiosos, es necesario que se cumplan los principios fundamentales del entrenamiento físico, y la intensidad de los ejercicios es un factor esencial. Actualmente se considera que mantener y promover la salud cardiovascular requiere realizar ejercicios aeróbicos de intensidad moderada un mínimo de 30 min cinco días a la semana o de intensidad vigorosa 20 min tres veces por semana. Puede considerarse el empleo de ejercicios isométricos o de resistencia aun en pacientes con insuficiencia cardiaca debidamente seleccionados y supervisados, Rivas, (2011).

Dicho beneficio se observa en ambos sexos y es mayor cuanto mayor es el volumen o la intensidad del ejercicio físico. Para obtener dichos beneficios, debe realizarse ejercicio aeróbico moderado durante un mínimo de 30 minutos, 5 días por semana, o ejercicio intenso durante un mínimo de 20 minutos, 3 días por semana. Se recomienda añadir un mínimo de 2 días no consecutivos cada semana para practicar 8-10 ejercicios que desarrollen la fuerza de la mayor parte de grupos musculares (brazos, hombros, tórax, abdomen, espalda, caderas y piernas), con 10-15 repeticiones de cada ejercicio. También es recomendable dedicar 2 sesiones de 10 minutos a la 
semana para realizar 8-10 ejercicios que mantengan la flexibilidad de la mayor parte de grupos de músculos y tendones. El ejercicio físico puede comportar lesiones del aparato locomotor y un riesgo cardiovascular, pero el beneficio supera al riesgo, Subirats et al, (2012).

La práctica regular de ejercicio físico es una recomendación establecida para prevenir y tratar los principales factores de riesgo cardiovascular modificables, como la diabetes mellitus, la hipertensión y la dislipemia. Realizar actividad física de intensidad moderada durante un mínimo de 30 min 5 días por semana o de intensidad alta durante un mínimo de 20 min 3 días por semana mejora la capacidad funcional y se asocia a reducciones en la incidencia de enfermedad cardiovascular y mortalidad. Cordero et al, (2014).

Se ha mostrado que llevar una vida físicamente activa implica muchos Beneficios para la salud: disminuye el riesgo de mortalidad enfermedades cardiovascular, previene y / o retrasa la desarrollo de la hipertensión arterial, mejora la perfil lipídico sanguíneo (reduce triglicéridos y aumenta el colesterol HDL), disminuye el riesgo de padecen diabetes tipo 2, e incluso ciertos tipos De cánceres (colon, cáncer de mama), mejora el cuerpo. Control de peso (prevención y tratamiento del sobrepeso y obesidad), ayuda a optimizar y mantener el fuerza y resistencia muscular, etc., Varela et al. (2013).

El ejercicio físico induce adaptaciones fisiológicas cardiovasculares que mejoran el rendimiento físico, y solo en casos extremos pueden conducir a un riesgo aumentado de complicaciones asociadas al ejercicio físico. La incidencia de muerte súbita o complicaciones graves durante la práctica de ejercicio físico es muy baja, se concentra en las personas con cardiopatías o con adaptación cardiaca muy patológica al ejercicio y la mayoría de estos casos los pueden detectar unidades de cardiología o profesionales bien instruidos, Cordero et al, (2014). 
La práctica regular de ejercicio físico a una intensidad ligera-moderada induce una serie de adaptaciones que producen los beneficios para la salud. Diferentes estudios han mostrado una relación inversa entre ejercicio habitual y riesgo de enfermedad coronaria, eventos cardiacos y muerte. El ejercicio mejora el perfil lipídico y el control de la glucemia, reduce o previene la hipertensión arterial, la obesidad y el estrés, mejora la forma física y aumenta la longevidad. Sin embargo, la mayoría de las evidencias de sus beneficios se extraen de estudios observacionales, y aunque el consumo máximo de oxígeno y el tiempo de ejercicio durante la prueba de esfuerzo son potentes predictores de mortalidad, no hay acuerdo en la cantidad y la intensidad de la actividad física necesaria en prevención primaria y secundaria, Boraita, A., (2009).

El ejercicio físico o la actividad física practicados de manera regular y planificado específicamente para personas mayores, está asociado con un menor riesgo de morbilidad y mortalidad y con una mejora en la calidad de vida de esta población, secundario al efecto protector cardiovascular que genera, el cual disminuye el riesgo de sufrir un infarto de miocardio y de desarrollar diabetes tipo 2, Landinez, et al. (2012).

También debe considerarse que los deportes de competición representan uno de los fenómenos culturales más interesantes de nuestra sociedad. Gracias básicamente a los medios de comunicación masiva e iniciativas privadas y patrocinio, numerosos eventos deportivos. Se han convertido en grandes entretenimientos. En este sentido, propiamente dicho. Alimentación e hidratación y entretenimientos sociales masivos. Son esenciales para adaptarse a optimizar los entrenamientos físico, rendimiento, y acelerar el proceso de recuperación. Y siempre tener en cuenta que un deporte será practicado. A nadie le gusta, mientras que llevar una vida activa es un principio ineludible para todo ciudadano. Varela et al. (2013). 
Se ha denunciado que muchos de los estudios sobre actividad física actuales no incluyen una clara recomendación sobre la cantidad de actividad física que se debe prescribir a la población, y menos aún si se desea personalizar la recomendación en función de los potenciales beneficios que se desea obtener, Schoeller, (2003).

\section{La prescripción del ejercicio físico o actividad deportiva}

La prescripción de ejercicio físico es útil especialmente para prevenir la mortalidad prematura de cualquier causa, la cardiopatía isquémica, la enfermedad cerebrovascular, la hipertensión arterial, el cáncer de colon y mama, la diabetes tipo 2, el síndrome metabólico, la obesidad, la osteoporosis, la sarcopenia, la dependencia funcional y las caídas en ancianos, el deterioro cognitivo, la ansiedad y la depresión, Subirats et al, (2012).

Consideraciones generales al momento de recomendar la actividad física, con especial énfasis en aspectos médico-deportivos. Algunos principios fundamentales son válidos para el entrenamiento muscular en cualquier situación. Factores como la edad, el sexo y el medio ambiente deben ser considerados siempre al iniciar un programa de entrenamiento con cualquier tipo de paciente. Además de las enfermedades asociadas y su severidad, las alternativas de actividad física varían en forma importante de acuerdo con el sexo y la edad, Duperly y Anchique, (2014).

Contenidos de fuerza y competitividad pueden, por ejemplo, favorecer la adherencia en muchos varones jóvenes o de edades medias, mientras que actividades como el baile y la gimnasia, generalmente con importantes componentes artísticos y estéticos, suelen ser más apropiados para las mujeres a cualquier edad. El medio ambiente puede ser determinante. Mientras que en muchas situaciones el ejercicio al aire libre y en contacto con la naturaleza pareciera ideal, los centros de 
gimnasia aeróbica y entrenamiento de fuerza en lugares seguros, con ambientes agradables y protegidos de las variaciones climáticas representan una alternativa ideal para la gran mayoría de habitantes de ciudades grandes, Duperly y Anchique, (2014).

En una persona que practica una actividad física y deportiva, con independencia de su nivel, las diferentes estrategias de exploración cardiológica deben permitir contraindicar acertadamente o readaptar una práctica deportiva que podría ser peligrosa para su salud, pero también, al contrario, no interrumpir una posible carrera deportiva o simplemente el equilibrio psicológico que proporciona, beneficio importante de la práctica deportiva: no hay que dudar, por lo tanto, en solicitar la opinión de un especialista, Ducardonnet, y Verdier, (2013).

Según ACSM, (2009), Molteni et al. (2004) y Pate et al. (1995), El Centro para el Control y Prevención de Enfermedades (CDC), el Colegio Americano de Medicina del Deporte (ACSM) y la Asociación Americana del Corazón (AHA) recomiendan la práctica de actividad física asociada a salud pública, enfatizando en una serie de aspectos que se resume a continuación:

1- Actividades Aeróbicas: se recomienda para personas de edad avanzada sanas realizar actividad física aeróbica que puede ser en base a dos modalidades.

A- De intensidad moderada, que significa un esfuerzo 5 a 6 en una escala de 1 a 10 , al menos 30 minutos al día, ente 5 a 7 días de la semana.

B- De alta intensidad, que implica un esfuerzo 7 a 8 en la escala de 1 a 10 , al menos 20 minutos al día, 3 veces por semana. 
La ACSM (26) recomienda en caso de realizar actividades de intensidad moderada una duración de 30 a 60 minutos al día. Sin embargo, está demostrado que no necesariamente la sesión debe ser continua. La persona puede realizar actividades de 10 minutos, tres o cuatro veces durante el día, evitando así cansancio. Lo importante es sumar entre 150 a 300 minutos a la semana para obtener los beneficios de esta actividad en la salud.

Si la actividad física es de alta intensidad o vigorosa, la recomendación es de 20 a 30 minutos al día, con un total de 75 a 150 minutos por semana.

2- Ejercicios de Resistencia o con pesas: deben ser realizados en series (1 a 3 ) que implican 8 a 12 repeticiones. Cada ejercicio debe trabajar un grupo muscular previamente definido. Para un trabajo integral y compensado se requiere entre 8 a 10 tipos de ejercicio diferentes. Pueden ser de intensidad moderada o vigorosa.

3- Flexibilidad: las actividades de al menos 10 minutos con el mayor número de grupos de músculos y tendones, de 10 a 30 segundos en 3 a 4 repeticiones de cada movimiento estático, todos los días en que se realicen actividades aeróbicas y de fuerza. Se debe realizar por lo menos dos días a la semana a una intensidad de 5 a 6 (en la escala de 0-10).

Recomendaciones especiales:

- $\quad$ Para los adultos mayores se recomienda incluir ejercicios de equilibrio.

- Para los adultos que tengan como objetivo perder peso o mantener el peso perdido, la recomendación es de acumular por lo menos de 60 a 90 minutos de actividad física moderada, por lo menos 5 días a la semana (de preferencia todos los días). 
- Para los niños y adolescentes la recomendación es de por lo menos 60 minutos de actividad de intensidad moderada a vigorosa, al menos 5 días de la semana, de preferencia todos, de forma continua o acumulada.

Lo más importante de este nuevo concepto es que cualquier actividad de la vida cotidiana es válida. Se enfatiza que la actividad física moderada puede ser realizada de forma continua o con intervalos, es decir, lo importante es que se acumulen durante el día por lo menos 30 minutos de actividad, en tres sesiones de 10 minutos o dos sesiones de 15 minutos. Para realizar AF no es necesario acudir a un gimnasio. Uno la puede realizar en casa incorporando las labores habituales como barrer, limpiar ventanas, lavar el auto, etc. En el trabajo (caminar como medio de transporte hacia o desde el trabajo, estacionar el coche más lejos, incluir 10 minutos de caminada a la hora del almuerzo, subir escaleras) y el tiempo libre (caminar, nadar, jugar deportes de pelota, o simplemente bailar) son aquellos que garantizan una vida sana y activa cuando el objetivo es promover la salud con la práctica regular de la actividad física, Matsudo, (2012).

El tipo de actividad física más recomendado en el mundo es la marcha. Caminar hace parte de nuestra vida diaria, no requiere de implementos o escenarios deportivos especiales, no tiene restricciones de horario y es accesible para la mayoría de la población. Desde el punto de vista médico tiene ventajas adicionales. Es fácil de dosificar, evitando así picos de intensidad frecuentes en otras actividades, no requiere de una gran capacidad cardiopulmonar, no produce una gran sobrecarga del aparato osteomuscular, ni se necesitan destrezas especiales en cuanto a coordinación, equilibrio, fuerza o velocidad. Duperly y Anchique, (2014).

Sin embargo, dependiendo de los objetivos y características de cada paciente es necesario recordar que el gasto calórico y por lo tanto el impacto metabólico de caminar es relativamente bajo, 
oscilando entre 200 y $300 \mathrm{kcal}$ por hora dependiendo del peso del paciente, la velocidad y las condiciones del terreno y del clima. Probablemente es el tipo de ejercicio ideal para comenzar un programa de acondicionamiento, pero puede ser insuficiente a medida que se buscan metas más ambiciosas. Por tratarse de una actividad relativamente monótona, la adherencia en algunas personas es limitada, Duperly y Anchique, (2014).

Actividad física y sedentarismo: de los padres, como ejemplo a seguir por sus hijos y de los propios niños, estimulando una vida activa con actividad física adecuada a cada una de las etapas de su maduración y limitando las actividades sedentarias, Chiesa et al. (2009).

\section{Problemas a causa del sedentarismo}

Una importante contribución al conocimiento de la relación entre sedentarismo y síndrome metabólico y representa un nuevo paso adelante en la investigación sobre la actividad física como determinante de la salud. Desafortunadamente aún queda camino por recorrer tanto en la definición precisa de un estilo de vida sedentario como en la promoción global e integral de la actividad física desde una perspectiva poblacional. La buena noticia es que al ser tan prevalente el sedentarismo, las oportunidades para desarrollar actividades preventivas son amplísimas, Cabrera et al. (2007).

La falta de actividad física es un problema de salud pública que se reconoce como un factor independiente de riesgo de enfermedad coronaria. El riesgo relativo de la inactividad es similar al de la hipertensión arterial, la hipercolesterolemia y el tabaquismo, por lo que el sedentarismo se asocia a un aumento simultáneo de las enfermedades cardiovasculares, Boraita, (2009).

El estilo de vida sedentario es un diagnóstico que presenta características definidoras de difícil mensuración y el IPAQ tiene el potencial para contribuir para la evaluación, más fidedigna,

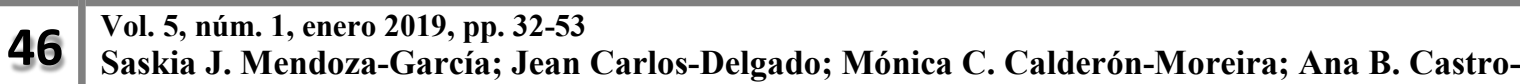
Soriano; Felipe A. Bajaña-Andrade; Marjorie L. Erazo-Villacreses
} 
de la presencia de la respuesta humana en cuestión. Finalmente, dada la amplitud y la complejidad del problema de sedentarismo, se alerta para la necesidad de alterar ese cuadro por medio de proveer y expandir las acciones que puedan influir en el comportamiento de las poblaciones, con la finalidad de promoción de la actividad física, en el sentido de disminuir la prevalencia de la hipertensión arterial, así como prevenir complicaciones en el estado de salud en consecuencia de ese agravio, Martins et al. (2009).

El estilo de vida sedentario, o mejor dicho, el inactivo. El estilo de vida es una característica común del estilo de vida de sociedades desarrolladas y es aún más pronunciado en los países mediterráneos del sur de Europa. Respecto a la población pediátrica, situaciones como la del menor nivel de seguridad vial y cívica ha hecho que el porcentaje de niños que viajan a la escuela a pie o en bicicleta o jugando en las calles, parques o los espacios públicos son más bajos. Este hecho podría ser incluido en el llamado "efecto canguro protector" que muchos padres adoptan impidiendo que sus hijos jueguen y corren por las calles “por su cuenta”. Varela et al. (2013).

Junto a los avances en las nuevas tecnologías hacen que las personas se involucren. Más y más en actividades de muy bajo consumo de energía. Durante su tiempo libre (consola de juegos, video juegos, internet, etc.). Debemos recordar que el comportamiento sedentario no es solo menor actividad física, pero un conjunto de actitudes individuales es que el hecho de estar sentado y/o tendido Abajo se convierte en el comportamiento postural predominante lo que implica un gasto energético muy limitado, Varela et al. (2013).

Los comportamientos sedentarios están presentes en muchos lugares y situaciones: el lugar de trabajo, el entorno escolar, Transporte, o tiempo libre y de ocio. El problema es que una serie de 
estudios realizados en los últimos años han demostrado que el sedentarismo o la inactividad física son un factor de riesgo para el desarrollo de muchas enfermedades crónicas, Varela et al. (2013).

Según Chiesa et al. (2009), el sedentarismo es causado por la vida moderna (computadores personales, video juegos, acceso a Internet, videocasete, DVD, etcétera) ha promovido la disminución de los tiempos de recreación física de los niños (juegos en la calle, plazas, etcétera). La prevención de esta situación requiere:

- Realizar actividad física 60 minutos, diarios o en días alternos;

- Limitar actividades sedentarias a menos de dos horas por día;

- Fomentar la participación en ejercicios recreativos y/o competitivos adecuados a cada paciente en particular.

\section{El riesgo de la enfermedad cardiovascular}

El riesgo cardiovascular y el riesgo coronario no son conceptos sinónimos. El primero incluye, además, la probabilidad de padecer enfermedad cerebrovascular y arterial periférica, pero en la práctica clínica habitual pueden emplearse indistintamente, ya que el riesgo coronario es una aproximación razonable del riesgo cardiovascular, y constituye uno de los aspectos más importantes y controversiales de la intervención terapéutica farmacológica, especialmente en el caso de la hipertensión arterial y la hiperlipidemia o dislipidemia, Vega, et al, (2011).

Entre los principales factores de riesgo causales de la ECV aterosclerótica destacan el tabaquismo, la hipertensión arterial (HTA), la hipercolesterolemia y la diabetes mellitus. Además, son muy frecuentes en la población, y por ello, dan cuenta de una parte muy importante de los casos de ECV en la población española, Banegas et al, (2003).

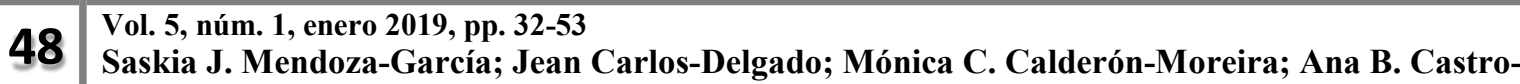
Soriano; Felipe A. Bajaña-Andrade; Marjorie L. Erazo-Villacreses
} 
Los factores de riesgo son aquellos signos biológicos o hábitos adquiridos que se presentan con mayor frecuencia en los pacientes con una enfermedad concreta. La enfermedad cardiovascular tiene un origen multifactorial, y un factor de riesgo debe ser considerado en el contexto de los otros. Los factores de riesgo cardiovascular, clásicos o tradicionales, se dividen en 2 grandes grupos: no modificables (edad, sexo y antecedentes familiares), y modificables (dislipidemia, tabaquismo, diabetes, hipertensión arterial, obesidad y sedentarismo), Vega, et al, (2011).

Estudios epidemiológicos han demostrado que la capacidad aeróbica se asocia en forma inversa y significativa a mortalidad cardiovascular y general, tanto en sujetos sanos, como en prevención secundaria. Esta relación es consistente y graduada e independiente de confusión. Basándose en la evidencia disponible, acerca de los beneficios del ejercicio en la reducción del riesgo de desarrollo y progresión de $\mathrm{ECV}$, se ha recomendado la práctica de actividad física regular como una estrategia para reducir su incidencia. Acevedo et al. (2013).

El riesgo cardiovascular se define como la probabilidad de padecer un evento cardiovascular en un determinado período, que habitualmente se establece en 5 ó 10 años, y su estratificación y cuantificación por el Médico de Familia, especialmente en los pacientes que no padecen enfermedad cardiovascular, es decir, en prevención primaria, es fundamental para establecer la intensidad de la intervención, la necesidad de instaurar tratamiento farmacológico y la periodicidad de las visitas de seguimiento. Guías de práctica clínica para el tratamiento de la hipertensión arterial, (2007).

Durante la realización de un ejercicio extenuante aumenta temporalmente el riesgo de infarto agudo de miocardio, el balance entre los riesgos y los beneficios es claramente favorable a éstos, aunque hay un umbral mínimo de gasto energético seminal necesario para disminuir el riesgo cardiovascular, Boraita, (2009). 
La importancia que hoy día tienen las enfermedades cardiovasculares en general y la angina de pecho (estable e inestable) en particular, así como el infarto agudo de miocardio, en los indicadores de mortalidad de la población. Las patologías cardiovasculares afectan mucho más a la población mayor, por lo que se vive una situación paradójica: la esperanza de vida es cada día mayor, al tiempo que es en las personas mayores donde se pueden observar más notoriamente dichas enfermedades; es, además, la población con menor actividad física, lo que es un buen elemento para contrastar la incidencia negativa de la enfermedad cardiovascular, Pelegrín, et al, (2009).

El argumento principal para la prevención es humanitario, no económico. En teoría, el éxito que se logre en la reducción de la tasa de incidencia global poblacional de la ECV debería disminuir los costes de los servicios sanitarios, ya que habría menos casos para tratar. En la práctica, sin embargo, los costes podrían aumentar debido al incremento continuo del gasto destinado a la investigación y al tratamiento de cada paciente, Rose, (1992).

La prevención cardiovascular se debe iniciar precozmente, tanto en niños con enfermedades que determinan situaciones especiales de alto riesgo como en aquellos que por su exposición a los factores de riesgo cardiovascular tienen un mayor riesgo de padecer enfermedad cardiovascular en su vida adulta. Los factores de riesgo comienzan a actuar desde edades tempranas, ya sea por exposición a hábitos no saludables de vida (mala alimentación, sedentarismo, tabaquismo) y/o a los clásicos factores de riesgo cardiovascular. La prevención se debe realizar en todas las edades, Chiesa et al. (2009). 


\section{Conclusiones.}

Para el paciente con diagnostico cardiovascular la prevención resulta ser una herramienta muy útil para el médico afín de establecer prioridades en la atención primaria, de igual manera, reconocer las diferencias de género en cuanto a los factores de riesgo, su avance y respuesta al tratamiento para efectuar las estrategias e intervenciones convenientes.

Como estrategia definitiva en la salud del paciente es un derecho imprescindible el ejercicio en las personas, como prevención secundaria, mismo que debe incluirse como programa de rehabilitación cardíaca debe mantenerse de manera indefinida y comprometerse a considerar como parte de los hábitos saludables de los pacientes, vale resaltar la importancia de la actividad física, como elemento fundamental para que las personas mayores se puedan mantener en un estado de salud óptimo; otra herramienta fundamental es la educación así como la alimentación puesto que como prevención permitirá evitar complicaciones que puedan afectar la esperanza de vida.

Es de primordial importancia considerar que nos enfrentamos en una época donde la actividad física es una herramienta indispensable para reducir las preocupaciones de los pacientes a fin de que pueda responder a todas las necesidades de los pacientes y afianzar un estado de vida con mejor calidad.

\section{Referencias Bibliográficas.}

ACSM, (2009), American College of Sports Medicine (ACSM) Position stand on exercise and physical activity for older adults. Med Sie Sports Exerc.

Acevedo, M., Krämer, V., Bustamante, M., Yáñez, F., Guidi, D., Corbalán, R., Godoy, I., Vergara, I., Jalil, J. y Fernández, M., (2013), Rehabilitación cardiovascular y ejercicio en prevención secundaria, Rev Med Chile 2013, 141: 1307-1314.

Banegas, J., Rodríguez, F., Graciani, A., Villar, F. y Herruzo, R., (2003), Mortality attributable to cardiovascular risk factors in Spain, Eur J Clin Nutr., 57: S18-21. 
Boraita, A., (2009), Ejercicio, piedra angular de la prevención cardiovascular, Servicio de Cardiología. Centro de Medicina del Deporte. Consejo Superior de Deporte. Revista Española de Cardiología, Volume 61, Issue 5, Pages 514-528 Madrid. España. https://doi.org/10.1157/13119996Get rights and content

Cordero, A., Masiá, M., Galve, E., (2014), Ejercicio físico y salud, Revista Española de Cardiología (English Edition), Volume 67, Issue 9, Pages 748-753, https://doi.org/10.1016/j.recesp.2014.04.007Get rights and content

Chiesa, P., Gambetta, J., Dutra, S., (2009), Prevención cardiovascular desde la edad pediátrica, Revista uruguaya de cardiología, Volumen $24 \mathrm{~N}^{\circ} 2,105-111$

Christmas, C. y Andersen, R., (2000), Exercise and older patients: guidelines for the clinician., J Amer Geriatrics Soc., 48: 318-24.

Ducardonnet, A. y Verdier, J., (2013), Corazón y deporte, EMC - Tratado de Medicina, Volume 17, Issue 3, pages 1-9, https://doi.org/10.1016/S1636-5410(13)64525-0Get rights and content

Duperly, J. y Anchique, C., (2014), Actividad física y prevención cardiovascular, texto de medicina interna: sección II, Cardiología, Capítulo 20 http://johnduperly.com/wpcontent/uploads/2014/03/JOHN-DUPERLY-ACMI-ACTIVIDAD-FISICA-REVENCIONCARDIOVASCULAR.pdf

Erhardt, L., (2007), Rationales for multiple risk interventions: the need to move from theory to practice, Vasc Health Risk Manag; 3(6): 985-97: Disponible en: http://www.ncbi.nlm.nih.gov/pmc/articles/PMC2350141/

Landinez, N., Contreras, K. y Castro, Á., (2012), Proceso de envejecimiento, ejercicio y fisioterapia, Revista Cubana de Salud Pública, 38(4): 562-580

Matsudo, S., (2012), Actividad física: pasaporte para la salud, Rev. Med. Clin. CONDES - 23(3) 209-217

Molteni, R., Zheng, J., Gómez, F., Twiss, J., (2004), Voluntary Exercise increases axonal regeneration from sensory neurons, Proc Natl Acad Sci U S A, 101(22): 8473-8

Murray, C. y López, A., (2002), Assessing health needs: the Global Burden of Disease Study. En: Detels R, McEwen J, Beaglehole R, Tanaka H, editors. Oxford textbook of public health. 4th ed. Oxford: Oxford University Press, 243-254.

OMS, (2007), Derecho a la Salud. Nota descriptiva No 323. Organización Mundial de la Salud, Centro de Prensa, Disponible en la siguiente Dirección Electrónica: http://www.who.int/mediacentre/factsheets/fs323/es/index.html [ Links ]

Pate, R., Pratt, M., Blair, S., et al. (1995), Physical activity and public health, A recommendation from the Center for Disease Control and Prevention and the American College of Sports Medicine, JAMA, 273(5): 402-7. 
Pelegrín, A., García, F. y Garcés, E., (2009), Prevención de los trastornos cardiovasculares: una propuesta de actuación desde la actividad física y el entrenamiento psicológico, Psicología y Salud, Vol. 19, Núm. 2: 261 - 269

Rivas, E., (2011), El ejercicio físico en la prevención la rehabilitación cardiovascular, Revista Española de Cardiología Suplementos, Volume 11, Supplement 5, Pages 18-22 https://doi.org/10.1016/S1131-3587(11)15004-9Get rights and content

Rose, G., (1992), The strategy of preventive medicine, New York: Oxford University Press.

Serra, L., de Cambra, S., Saltó, E., Roura, E., Rodríguez, F., Vallbona, C. y Salleras, L., (1994), Consejo y prescripción de ejercicio físico, Medicina Clínica, Vol. 102., Suplemento 1: 100108, Barcelona.

Simonetti, J., Batista, L., Carvalho L., (2002), Hábitos de saúde e fatores de risco em pacientes hipertensos, Rev Latino- am Enfermagem, 10(3): 415-22.

Schoeller, D., (2003), But how much physical activity? Am J Clin Nutr., 78: 669-70.

Subirats, E., Subirats, G., Iñigo, M., (2012), Prescripción de ejercicio físico: indicaciones, posología y efectos adversos, Medicina Clínica, Volume 138, Issue 1, 21, Pages 18-24 https://doi.org/10.1016/j.medcli.2010.12.008Get rights and content

Varela, G., Alguacil, L., Alonso, E., Aranceta, J., Ávila, J., Aznar, S., Belmonte, S., et al. (2013), Obesidad y sedentarismo en el siglo XXI: ¿Qué se puede hacer y qué se debe hacer?, Nutr Hosp 2013; 28 (Supl. 5): 1-12 ISSN, (Versión electrónica): 1699-5198

Varo, J. y Martínez, M., (2007), Los retos actuales de la investigación en actividad física y sedentarismo, Rev Esp Cardiol., 60(3): 231-3

Varo, J.J.; Martínez-González, M.A.; De Irala-Estévez, J.; Kearney, J; Gibney, M. \& Martínez, J.A. (2003), Distribution and determinants of sedentary lifestyles in the European Union. International Journal of Epidemiology, 32, 138-146.

Vega, J., Guimará, M. y Vega, L., (2011), Riesgo cardiovascular, una herramienta útil para la prevención de las enfermedades cardiovasculares, Revista Cubana de Medicina Integral: 27(1)91-97 http://scielo.sld.cu

WHO, (2002), World Health Report (2002). Geneva: World Health Organization, 2002. 